\title{
M-Leaning en apoyo a la Carrera de Ecoturismo modalidad virtual y presencial
}

Carlos Francisco Agurcia Ramos

carlos.agurcia@unah.edu.hn

Centro Regional del Litoral Atlantico - UNAH (CURLA-UNAH)

\section{Resumen}

Las innovaciones pedagógicas y tecnológicas del presente amplían los escenarios de aprendizaje, de manera que facilitan el acceso a las actividades educativas en cualquier momento y lugar. Desde este enfoque y para resolver problemas como el acceso a contenidos educativos en línea en tiempo real, se llevó a cabo el proyecto de innovación educativa Desarrollo de una aplicación para los contenidos del Seminario de Relaciones Humanas de la Carrera de Ecoturismo en línea de la UNAH.

El producto final de este proyecto es una aplicación móvil que de manera asincrónica permite acceder a los contenidos de la asignatura Seminario de Relaciones Humanas.

En este artículo se presentan las distintas fases de desarrollo de este proyecto, sus principales resultados y el beneficio para los estudiantes de la licenciatura de Ecoturismo en línea que tienen dificultades de conectividad y acceso al equipo de cómputo para ingresar a la plataforma educativa y estudiar los contenidos de aprendizaje en forma asincrónica.

\section{Palabras claves:}

$M$-Learning, aplicación informática, relaciones humanas y App

\section{Abstract}

The pedagogical and technological innovations of the present extend the learning scenarios, generating facilities to access the educational activities at any time and place. Under this approach and to solve problems such as access to online educational content in real time, the educational innovation project "Development of an app for the contents of the Human Relations seminar of the Ecotourism career in UNAH line.

The final product of this project is a mobile application that allows asynchronous access to the contents of the subject Human Relations Seminar. The different phases of development of this project, its main results and the benefit for students of the online ecotourism degree who have difficulties of connectivity and access to computer equipment to access the educational platform and study the learning contents in an asynchronous form are presented in this article.

\section{Keywords:}

M-Learning, computer application, human relations and Apps

\section{Introducción.}

El uso de teléfonos móviles "inteligentes" es cada vez más común, y dentro de estos los dispositivos con sistema operativo Android son los más utilizados (Gutierrez, 2015).

Al conversar con docentes y padres de familia es común escucharlos decir que los jóvenes están "inmersos" en sus dispositivos móviles y difícilmente se les puede reducir el tiempo de uso. Pero desde un enfoque educativo innovador lo que sí es posible es aprovechar el potencial de estas tecnologías y las habilidades de los estudiantes para usarlas 
como herramientas didácticas para el aprendizaje; es decir, colocar parte del proceso educativo en estos dispositivos móviles.

Una aplicación móvil es un programa que puede ser descargado y al que se puede acceder directamente desde el celular u otro dispositivo móvil, como una Tablet, de acuerdo con la Comisión Federal del Comercio del gobierno de los Estados Unidos de América (2011).

Este tipo de aplicaciones se utilizan para desarrollar tareas específicas, algunas, por ejemplo, sirven como calculadora o calendarios, otras permiten acceder a redes sociales, también pueden ser procesadores de texto $\mathrm{u}$ editores de hojas de cálculo, entre otras.

Según IBM Corporation (2012) hay tres tipos de aplicaciones móviles:

a. Aplicaciones nativas. Son aquellas diseñadas bajo un lenguaje del entorno específico, con la ventaja de ser muy fluidas en determinada plataforma, pero no pueden ejecutarse en otras plataformas.

b. Aplicaciones web. Estas se ejecutan desde el navegador web del dispositivo, su ventaja principal es que pueden ser ejecutas desde cualquier dispositivo con cualquier sistema operativo; sin embargo, presentan la desventaja de que requieren una conexión permanente al internet.

c. Aplicaciones híbridas. Son desarrolladas para funcionar en dos o más sistemas operativos, pero su creación tiende a ser más costosa.
El uso de móviles y de aplicaciones móviles es cada vez más frecuente en el mundo académico y laboral, ya que la producción y utilidad de estas - tanto en su versión de código abierto como privado - inunda cada vez más el mercado laboral público y privado. Los centros educativos también aprovechan estos desarrollos para acercar más la academia al mundo de trabajo real.

Desde este contexto global y al considerar la situación socioeconómica de Honduras, específicamente de los estudiantes universitarios - que en un porcentaje significativo se ven en la necesidad de trabajar para costear sus estudios universitarios-, es que la UNAH visualiza soluciones educativas que permitan acercar más el conocimiento y la universidad a las personas que así lo demandan. Es decir, poner a disposición de la población joven —que aún no cuenta con educación universitaria-, modalidades educativas flexibles que aprovechen el potencial educativo de las tecnologías actuales. Con esto en perspectiva, la UNAH oferta desde el año 2010 carreras en modalidad virtual a través de los centros regionales siguientes: la UNAH en el Valle de Sula (UNAH-VS), el Centro Universitario Regional de Occidente (CUROC), y el Centro Universitario Regional del Litoral Atlántico (CURLA). En el CURLA la oferta académica virtual se inició en el año 2014, con las carreras de Licenciatura en Ecoturismo y el Técnico en
Microfinanzas, para atender la demanda de educación superior en Roatán, Islas de la Bahía. Para ello se creó el Telecentro UNAH-CURLA/Roatán, lo que ha permitido que los habitantes de esa isla tengan acceso a educación superior de calidad.

Como parte de la constante interacción y comunicación con los estudiantes de la modalidad virtual del Telecentro del CURLA en Roatán se identificaron algunas dificultades con las que se enfrentan los alumnos de la modalidad virtual debido a la realidad geográfica de esa zona, entre ellas: difícil acceso a una computadora u otro dispositivo con conexión a Internet durante el día y por tiempos prolongados.

Esa situación les impide conectarse por tiempos prolongados a sus aulas de clases virtuales, acceder a los contenidos en tiempo real y - por sus horarios laborales-, asistir al Telecentro, donde tienen acceso a computadoras e Internet de forma gratuita.

Ante esta situación se propuso la creación de una aplicación móvil, compatible con dispositivos con sistema operativo Android, que permitiera al estudiante de la licenciatura de Ecoturismo en línea, acceder a los contenidos temáticos y material educativo de la asignatura "Seminario de Relaciones $\mathrm{Hu}^{-}$ manas" sin necesidad de estar conectado a Internet, excepto al momento de instalar la aplicación y descargar los contenidos. 


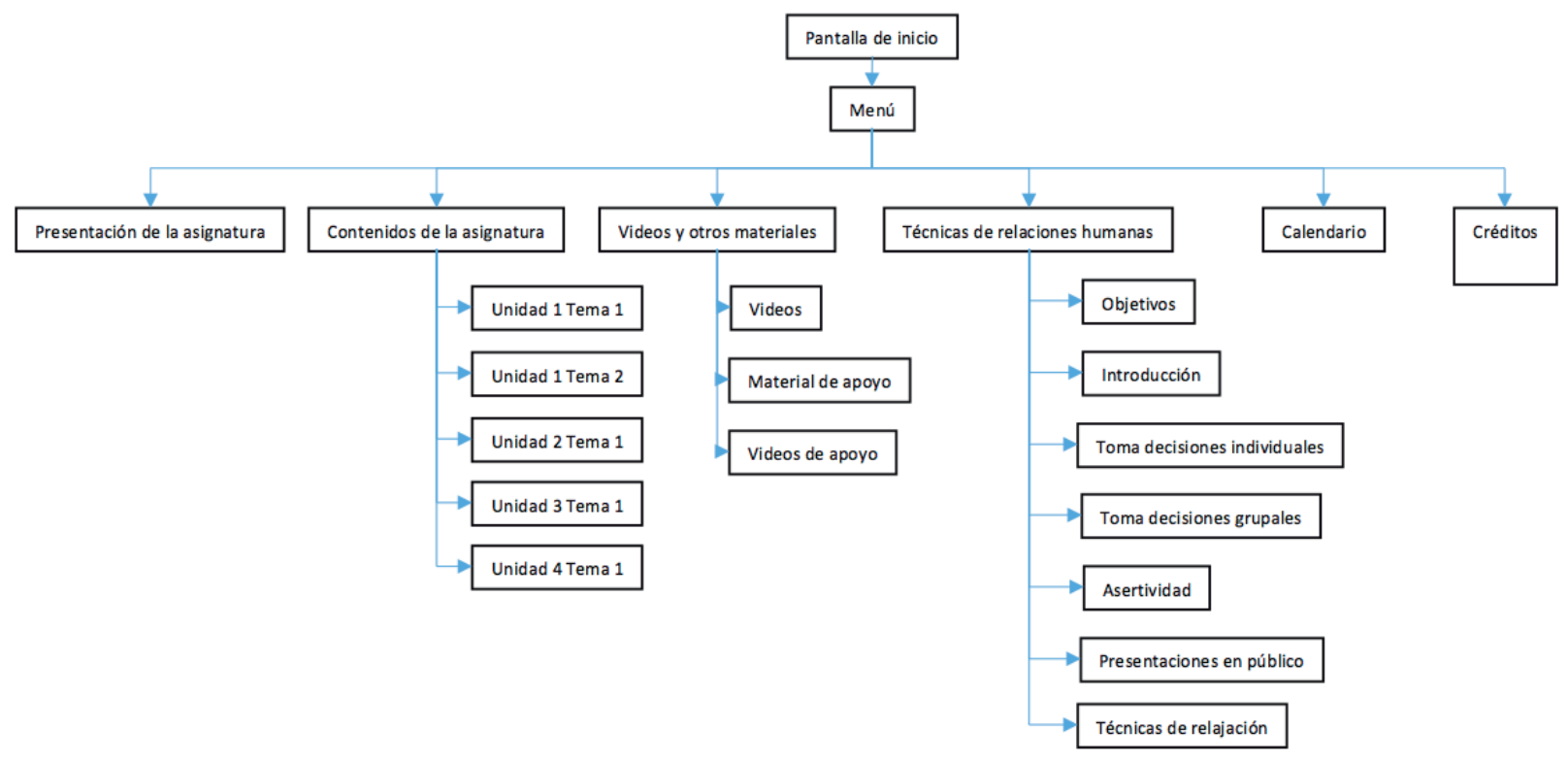

Figura 1. Esquema de la aplicación

Esto facilita a los estudiantes acceder y estudiar los temas en cualquier momento libre que dispongan en el día, mejora su rendimiento en la asignatura y les permite realizar una serie de ejercicios prácticos para el desarrollo de habilidades sociales.

\section{Fundamento teórico M-Learning}

Con la revolución tecnológica del mundo actual, los procesos educativos tienen la posibilidad de salirse del aula tradicional, es decir de las cuatro paredes físicas y del docente como el único transmisor de información y conocimiento. El Internet y las computadoras posibilitan por un lado la distribución de todo tipo de información y conocimiento en distintos formatos, asimismo la comunicación e interacción de todos los actores del proceso educativo. En este ámbito se popularizan los sistemas móviles (teléfonos inteligentes y tabletas) que están generando una nueva revolución, se han convertido en herramientas fundamentales para la educación. Este tipo de aprendizaje que se apoya en esta tecnología se denomina Mobile Learning o M-Learning.

Es decir, el M-learning "es el aprendizaje que se produce cuando el alumno no se encuentra en una ubicación fija y predeterminada; o de aprendizaje que se produce cuando el alumno se aprovecha de las oportunidades de aprendizaje que ofrecen las tecnologías móviles" (SCOPEO, 2011).

Actualmente el M-Learning se utiliza principalmente como apoyo a otros tipos de educación (presencial, a distancia, b-learning, e-learning y MOOCS) y en la mayoría de los casos requiere que la persona disponga permanentemente conexión a Internet en su dispositivo móvil. Según la UNESCO (2013) el m-learning tiene por lo menos las siguientes ventajas:

a) Mayor alcance e igualdad de oportunidades en la educación.

b) Facilidad para el aprendizaje personalizado.

c) Respuesta y evaluación inmediatas.

d) Aprendizaje en cualquier momento y lugar.

e) Empleo productivo del tiempo pasado en el aula.

f) Creación de nuevas comunidades de educandos.

g) Apoyo al aprendizaje en lugares concretos.

h) Mejora del aprendizaje continuo.

i) Vínculo entre la educación formal y no formal.

j) Mínimos trastornos para el aprendizaje en las zonas de conflicto y de desastre. 
Tabla 1. Habilidades sociales por desarrollar y sus componentes

\begin{tabular}{ll}
\multicolumn{1}{c}{ Habilidad } & \multicolumn{1}{c}{ Contenidos } \\
\hline Toma de decisiones en equipo & - Lluvia de ideas \\
& - Grupo nominal \\
& - Diagramas \\
Asertividad & - Comunicación asertiva \\
& - Negación asertiva \\
Hablar en publico & - Elaborar un material para presentar \\
& - Organizar las ideas de presentación \\
& - Estrategias para hablar en público \\
Toma de decisiones & - Valoración de la situación \\
& - Identificación de opciones \\
& - Valoración de opciones \\
& - Toma de decisión
\end{tabular}

k) Apoyo a los educandos con discapacidad.

1) Mejora de la comunicación y la administración.

m)Máxima eficacia en función de los costos.

\section{Metodología utilizada}

Esta aplicación se desarrolló con la finalidad de permitir al estudiante acceder al contenido y a los recursos sin necesidad de acceso a internet, excepto al momento de descargar la aplicación y los videos de la asignatura de Seminario de Relaciones Humanas de la carrera de Ecoturismo e incursionar en el m-learning. Se procedió, de acuerdo con el modelo de cinco etapas planteado por Gasca, Camargo y Delgado (2013):

Etapa de análisis: en esta etapa se identifican las necesidades de los usuarios y desarrolla una idea del producto que pueda satisfacerlas, para esto se identificó mediante comentarios con estudiantes y docentes que un gran problema expresado por los estudiantes de la modalidad a distancia virtual en Roatán es el acceso a Internet para poder acceder al contenido de la asignatura y el tiempo para poder ubicarse en algún lugar para poder leer los contenidos. Con base en esas necesidades surgió la idea de poner a su disposición una aplicación para teléfono móvil que ya incluya los contenidos, con lo que se resuelve el primer problema, y que se pudiera acceder a ella en cualquier momento libre, esto resuelve el segundo.

Etapa de diseño: en esta etapa se diseña la estructura de la aplicación, para esto se creó la estructura que se presenta en la Figura 1.

Etapa de desarrollo: en este momento se desarrolló el software, para lo cual se recurrió al recurso gratuito de Mobincube, que es un generador en línea de aplica- ciones nativas para dispositivos móviles que permite crear aplicaciones básicas a personas con nulos o limitados conocimientos sobre programación.

Se generó una aplicación para dispositivo móvil con sistema operativo Android llamada "Relaciones Humanas" disponible para los estudiantes del telecentro UNAH CURLA-Roatán. Esta aplicación incluye:

- La opción de descarga de los contenidos de la asignatura "Seminario de Relaciones Humanas" y acceder a ellos de manera asincrónica.

- Opción de descargar videos y materiales de apoyo de la asignatura y acceder sin $\mathrm{co}^{-}$ nexión a internet.

- Opción de descarga de instrucciones que describen paso a paso el desarrollo de métodos y técnicas que facilitan desarrollar ejercicios para habilidades sociales detalladas en Tabla No. 1. 
Tabla 2. Evaluación de la aplicación.

\begin{tabular}{|cccc}
\hline Ventaja & $\begin{array}{c}\text { Puntuación } \\
\text { dada por el } \\
\text { estudiante }\end{array}$ & Puntuación \\
$\begin{array}{cccc}\text { Mayor alcance e igual- } \\
\text { dad de oportunidades en } \\
\text { la educación }\end{array}$ & 3.5 & $\begin{array}{c}\text { Mejora del aprendizaje } \\
\text { continuo }\end{array}$ & 3.5 \\
$\begin{array}{c}\text { Facilidad para el apren- } \\
\text { dizaje personalizado }\end{array}$ & 3.9 & $\begin{array}{c}\text { Vínculo entre la educa- } \\
\text { ción formal y no formal }\end{array}$ & 3.7 \\
$\begin{array}{c}\text { Aprendizaje en cualquier } \\
\text { momento y lugar }\end{array}$ & 3.8 & $\begin{array}{c}\text { Aprendizaje en las zonas } \\
\text { de conflicto y de desas- } \\
\text { tre }\end{array}$ & 3.5 \\
$\begin{array}{c}\text { Empleo productivo del } \\
\text { tiempo pasado en el aula }\end{array}$ & 3.7 & $\begin{array}{c}\text { Apoyo a los educandos } \\
\text { con discapacidad }\end{array}$ & 3.5 \\
$\begin{array}{c}\text { Apoyo al aprendizaje en } \\
\text { lugares concretos }\end{array}$ & 3.3 & $\begin{array}{c}\text { Máxima eficacia en fun- } \\
\text { ción de los costos }\end{array}$ & 4 \\
\hline
\end{tabular}

Etapa de prueba de funcionamiento: para lo cual se descargó el archivo ".apk" un archivo que permite la instalación de la aplicación en el dispositivo móvil con sistema operativo Android y se instaló. Luego los estudiantes pudieron explorar la aplicación poniendo a prueba cada una de sus funciones.

Etapa de entrega: esta etapa se cumplió poniendo a disposición de los estudiantes la aplicación para su uso en la asignatura correspondiente. En vista que la asignatura no se sirvió en el Telecentro CURLA-Roatán, la validación de la aplicación se hizo con 6 estudiantes que cursaron la asignatura de Seminario de Relaciones Humanas en modalidad presencial durante el II PAC 2016 y 11 estudiantes que cursaron esta asignatura durante el III PAC 2016.
Se midió el impacto educativo de la aplicación a través de una encuesta de nivel de logro de la aplicación en 10 de las 13 ventajas del M-Learning según la UNESCO.

\section{Resultados.}

Se valoraron las 10 ventajas del $M$-Learning planteadas por la UNESCO, que se consideró que la aplicación desarrollada podría cumplir. Para ello se elaboró una encuesta de nivel de logro en la que los estudiantes podían elegir entre 4 opciones.

En la tabla 2 se puede observar la ponderación de los resultados obtenidos. Se puede apreciar que las 10 ventajas evaluadas tienen una puntuación superior a 3 puntos, lo que indica que los estudiantes dan una valoración alta al logro de la ventaja por la aplicación generada.
Dentro de los aspectos mejor valorados se puede observar la reducción de costos para el estudiante, ya que esta aplicación les evita la necesidad de adquirir materiales en físico, ya sea folletos, libros, artículos, etc., y también elimina la necesidad de acceder frecuentemente a internet para acceder a algún contenido en particular.

Luego se puede observar que los encuestados valoran el hecho que la aplicación les permite acceder a la información de la asignatura en cualquier momento y lugar, facilitándoles un ritmo personalizados de avance. También la consideran útil para facilitar la información de la asignatura a aquellas personas que residen en zonas peligrosas, que trabajan y estudian, personas con condiciones especiales y que en muchos momentos se les puede dificultar trasladarse a la universidad. Finalmente se recogieron co- 
mentarios y sugerencias de los estudiantes en relación con la aplicación. Los alumnos le dan una alta valoración a la facilidad de uso de la aplicación para el acceso a los contenidos y para el desarrollo de las actividades para las habilidades sociales.

Sugieren el desarrollo de este tipo de aplicaciones para otras asignaturas y se amplíe el uso para sistema operativo IOS.

\section{Conclusiones}

El desarrollo de la aplicación dirigida para los alumnos del Telecentro UNAH CURLA-Roatán, que cursen la asignatura de "Seminario de Relaciones $\mathrm{Hu}$ mana”, logró el objetivo planteado. Ya que fue posible el uso de la aplicación generada para la descarga de los contenidos y recursos educativos en los aparatos móviles Android y luego hacer su uso de forma asincrónica.

Con el uso que hicieron los estudiantes de esta aplicación, se constata el alto potencial de habilidad tecnológica de los alumnos, quienes reaccionaron de manera positiva al uso de la herramienta que se les posibilitó para usar en sus teléfonos móviles. Se debe tener presente que un alto porcentaje de los alumnos matriculados en la UNAH son nativos digitales; es decir, estudiantes jóvenes que en su mayoría son menores de 30 años.

Los resultados de la utilización de la aplicación en la asignatura "Seminario de Relaciones Hu- manas" muestran que los estudiantes valoran a la aplicación de manera positiva, principalmente por factores como: Reducción de costos, facilidad en aprendizaje personalizado, facilidad de acceder a los contenidos en diferentes lugares y momentos y que el tiempo en el aula se puede utilizar de manera más productiva. Originalmente la aplicación fue pensada para el apoyo de la educación en línea, sin embargo, al analizar los resultados se pretende utilizarla, también, con los estudiantes en la modalidad presencial, lo que demostró un mejor aprovechamiento de la asignatura y permite a los docentes enriquecer el espacio de aprendizaje con herramientas novedosas que facilitan el aprovechamiento del tiempo en el aula con actividades prácticas.

Se recomienda aprovechar la experiencia de la generación de esta aplicación, para el desarrollo de otros proyectos de innovación educativa similares con otras asignaturas. Actualmente existen varias plataformas de desarrollo de aplicaciones que presentan sus servicios de forma gratuita o en versiones pagas, un docente no necesita tener un alto perfil tecnológico, ni conocimientos de programación para poder desarrollar una aplicación de este tipo. Existen videotutoriales sencillos de fácil acceso que explican el proceso.

\section{Referencias}

Comisión Federal del Comercio del gobierno de los
Estados Unidos de América. (2011). Alerta en linea. Aplicaciones móviles: Qué son y cómo funcionan: Recuperado de: https:// www.alertaenlinea.gov/ articulos/s0018-aplicaciones-m\%C3\%B3viles-qu\%C3\%A9-son-y-c\%C3\%B3mo-funcionan

Gutierrez, O. (2015). Apple vende más, pero Android sigue dominando: estudio. CNET en Español. Recuperado de http://www.cnet.com/ es/noticias/apple-google-android-ios-participacion-mercado-2015/

IBM Corporation. (2012). El desarrollo de aplicaciones moviles nativas, web o hibridas. Recuperado de ftp://ftp.software.ibm. com/la/documents/gb/ commons/27754_IBM_ WP_Native_Web_or_hybrid_2846853.pdf

SCOPEO (2011). M-learning en España, Portugal y América Latina, Noviembre de 2011. Monográfico SCOPEO, no 3. Recuperado de: http:// scopeo.usal.es/wp-content/uploads/2013/04/scopeom003.pdf

UNESCO (2013). Directrices de la UNESCO para las políticas de aprendizaje móvil. Paris, Francia: UNESCO. 